\title{
Information loss in an optimal maximum likelihood decoding
}

\author{
Inés Samengo \\ Centro Atómico Bariloche and Instituto Balseiro \\ (8400) San Carlos de Bariloche, Río Negro, Argentina
}

\begin{abstract}
The mutual information between a set of stimuli and the elicited neural responses is compared to the corresponding decoded information. The decoding procedure is presented as an artificial distortion of the joint probabilities between stimuli and responses. The information loss is quantified. Whenever the probabilities are only slightly distorted, the information loss is shown to be quadratic in the distortion

PACS numbers: 07.05.Mh,87.10.+e,87.19.Dd,89.70.+c
\end{abstract}

Understanding the way external stimuli are represented at the neuronal level is one central challenge in neuroscience. An experimental approach to this end (Optican and Richmond 1987, Eskandar et al. 1992, Tovée et al. 1993, Kjaer et al. 1994, Heller et al. 1995, Rolls et al. 1996, Treves et al. 1996, Rolls et al. 1997, Treves 1997, Rolls and Treves 1998, Rolls et al. 1998) consists in choosing a particular set of stimuli $s \in \mathcal{S}$ which can be controlled by the experimentalist, and exposing these stimuli to a subject whose neural activity is being registered. The set of neural responses $r \in \mathcal{R}$ is then defined as the whole collection of recorded events. It is up to the researcher to decide which entities in the recorded signal are considered as events $r$. For example, $r$ can be defined as the firing rate in a fixed time window, or as the time difference between two consecutive spikes, or the $k$ first principal components of the time variation of the recorded potentials in a given interval, and so forth.

Once the stimulus set $\mathcal{S}$ and the response set $\mathcal{R}$ have been settled, the joint probabilities $P(r, s)$ may be estimated from the experimental data. This is usually done by measuring the frequency of the joint occurrence of stimulus $s$ and response $r$, for all $s \in \mathcal{S}$ and $r \in \mathcal{R}$. The mutual information between stimuli and responses reads (Shannon 1948)

$$
I=\sum_{s} \sum_{r} P(r, s) \log _{2}\left[\frac{P(r, s)}{P(r) P(s)}\right],
$$

where

$$
\begin{aligned}
& P(r)=\sum_{s} P(r, s) \\
& P(s)=\sum_{r} P(r, s) .
\end{aligned}
$$

The mutual information quantifies how much can be learned about the identity of the stimulus shown just by looking at the responses. Accordingly, and since $I$ is symmetrical in $r$ and $s$, its value is also a measure of the amount of information that the stimuli give about the responses. From a theoretical point of view, $I$ is the most appealing quantity characterizing the degree of correlation between stimuli and responses that can be defined. This stems from the fact that $I$ is the only additive functional of $P(r, s)$ ranging from zero (for uncorrelated variables) up to the entropy of stimuli or responses (for a deterministic one to one mapping) (Fano 1961, Cover and Thomas 1991).

However, even if formally sound, the mutual information has a severe drawback when dealing with experimental data. Many times, and specifically when analyzing data of multi-unit recordings, the response set $\mathcal{R}$ is quite large, its size increasing exponentially with the number of neurons sampled. Therefore, the estimation of $P(r, s)$ from the experimental frequencies may be far from accurate, specially when recording from the vertebrate cortex, where there are long time scales in the variability and statistical structure of the responses. The mutual information $I$, being a non linear function of the joint probabilities, is extremely sensitive to the errors that may be involved in their measured values. As derived in Treves and Panzeri (1995), Panzeri and Treves (1996) and Golomb et al. (1997), the mean error in calculating $I$ from the frequency table of events $r$ and $s$ is linear in the size of the response set. This analytical result has been obtained under the assumption that different responses behave independently. Although there are situations where such a condition does not hold (Victor and Purpura, 1997) it is widely accepted that the bias grows rapidly with the size of the response set.

Therefore, a common practice when dealing with large response sets is to calculate the mutual information not between $\mathcal{S}$ and $\mathcal{R}$, but between the stimuli and another set $\mathcal{T}$ each of whose elements $t$ is a function of the true response $r$, that is, $t=t(r)$ (Treves 1997, Rolls and Treves 1998). It is easy to show that if the mapping between $r$ and $t$ is one to one, then the mutual information between $\mathcal{S}$ and $\mathcal{R}$ is the same as the one between $\mathcal{S}$ and $\mathcal{T}$. However, for one to one mappings, the number of elements in $\mathcal{T}$ is the same as in $\mathcal{R}$. A wiser procedure is to choose a set $\mathcal{T}$ that is large enough not to lose the relevant information, but sufficiently small as to avoid significant limited sampling errors. One possibility is to perform a decoding procedure (Gochim et al. 1994, Rolls et al. 1996, Victor and Purpura 1996, Rolls and Treves 1998). In this case, $\mathcal{T}$ is taken to coincide with $\mathcal{S}$. To make this correspondence explicit, the set $\mathcal{T}$ will be denoted 
by $\mathcal{S}^{\prime}$ and its elements $t$ by $s^{\prime}$. Each $s^{\prime}$ in $\mathcal{S}^{\prime}$ is taken to be a function of $r$, and is called the predicted stimulus of response $r$. As stated in Panzeri et al. (1999), this choice for $\mathcal{T}$ is the smallest that could potentially preserve the information of the identity of the stimulus. The data processing theorem (Cover and Thomas, 1991) states that since $s^{\prime}$ is a function of $r$ alone, and not of the true stimulus $s$ eliciting response $r$, the information about the real stimulus can only be lost and not created by the transformation from $r \rightarrow s^{\prime}$. Therefore, the true information $I$ is always at least as large as the decoded information $I_{D}$, the latter being the mutual information between $\mathcal{S}$ and $\mathcal{S}^{\prime}[1]$. In order to have $I$ and $I_{D}$ as close as possible, it is of course necessary to choose the best $s^{\prime}$ for every $r$. The procedure consists in identifying which of the stimuli was most probably shown, for every elicited response. The conditional probability of having shown stimulus $s$ given that the response was $r$ reads

$$
P(s \mid r)=\frac{P(r, s)}{P(r)} .
$$

Therefore, the stimulus that has most likely elicited response $r$ is

$$
s^{\prime}(r)=\max _{s} P(s \mid r)=\max _{s} P(r, s) .
$$

By means of Eq. (5), a mapping $r \rightarrow s^{\prime}$ is established: each response has its associated maximum likelihood stimulus. Equation (4) provides the only definition of $P(s \mid r)$ that strictly follows Bayes' rule, so in this case, the decoding is called optimal. There are other alternative ways of defining $P(s \mid r)$ (Georgopoulos et al. 1986, Wilson and McNaughton 1993, Seung and Sompolinsky 1993, Rolls et al. 1996) some of which have the appealing property of being simple enough to be plausibly carried out by downstream neurons themselves. The purpose of this letter, however, is to quantify how much information is lost when passing from $r$ to $s^{\prime}$ using an optimal maximum likelihood decoding procedure.

In general, there are several $r$ associated with a given $s^{\prime}$. One may therefore partition the response space $\mathcal{R}$ in separate classes $\mathcal{C}(s)=\left\{r / s^{\prime}(r)=s\right\}$, one class for every stimulus. The number of responses in class $s^{\prime}$ is $N_{s^{\prime}}$. Of course, some classes may be empty. Here, the assumption is made that each $r$ belongs to one and only class (that is, Eq. (5) has a unique solution).

The joint probability of showing stimulus $s$ and decoding stimulus $s^{\prime}(r)$ reads

$$
P\left(s^{\prime}, s\right)=\sum_{r \in \mathcal{C}\left(s^{\prime}\right)} P(r, s),
$$

and the overall probability of decoding $s^{\prime}$,

$$
P\left(s^{\prime}\right)=\sum_{s} P\left(s^{\prime}, s\right)=\sum_{r \in \mathcal{C}\left(s^{\prime}\right)} P(r) .
$$

Clearly, with these definitions the decoded information

$$
I_{D}=\sum_{s} \sum_{s^{\prime}} P\left(s^{\prime}, s\right) \log _{2}\left[\frac{P\left(s^{\prime}, s\right)}{P\left(s^{\prime}\right) P(s)}\right]
$$

may be calculated, and has, in fact, been used in several experimental analyses (Rolls et al. 1996, Treves 1997, Rolls and Treves 1998, Panzeri et al. 1999). However, up to date, no rigorous relationship between $I$ and $I_{D}$ has been established. The derivation of such a relationship is the main purpose here.

When performing a decoding procedure, $r$ is replaced by $s^{\prime}$. Such a mapping allows the calculation of $P\left(s^{\prime}, s\right)$, after which any additional structure, which may eventually have been present in $P(r, s)$, is neglected. For example, if two responses $r_{1}$ and $r_{2}$ encode the same stimulus $s^{\prime}$ it becomes irrelevant whether, for a given $s$, $P\left(r_{1}, s\right)$ is much bigger that $P\left(r_{2}, s\right)$ or, on the contrary, $P\left(r_{1}, s\right) \approx P\left(r_{2}, s\right)$. The only thing that matters is the value of the sum of the two: their global contribution to $P\left(s^{\prime}, s\right)$. As a consequence, it seems natural to consider the detailed variation of $P(r, s)$ within each class, when estimating the information lost in the decoding.

In this spirit, and aiming at quantizing such a loss of information, $P(r, s)$ is written as

$$
P(r, s)=\frac{P\left[s^{\prime}(r), s\right]}{N_{s^{\prime}(r)}}+\Delta(r, s),
$$

where $\Delta(r, s)=P(r, s)-P\left[s^{\prime}(r), s\right] / N_{s^{\prime}(r)}$. Thus, the joint probability $P(r, s)$, which in principle may have quite a complicate shape in $\mathcal{R}$ space, is separated into two terms. The first one is flat inside every single class $\mathcal{C}\left(s^{\prime}\right)$, and the second is whatever needed to re-sum $P(r, s)$. It should be noticed that

$$
\sum_{r \in \mathcal{C}\left(s^{\prime}\right)} \Delta(r, s)=0
$$

for all $s$. Summing Eq. (9) in $s$,

$$
P(r)=\frac{P\left[s^{\prime}(r)\right]}{N_{s^{\prime}(r)}}+\Delta(r),
$$

where

$$
\Delta(r)=\sum_{s} \Delta(r, s)
$$

and

$$
\sum_{r \in \mathcal{C}\left(s^{\prime}\right)} \Delta(r)=0 .
$$

Replacing Eqs. (9) and (11) in the mutual information (11), one arrives at

$$
I=I_{D}+\sum_{r} \sum_{s} P(r, s) \log _{2}\left[\frac{P(r, s)}{Q(r, s)}\right]
$$


where

$$
Q(r, s)=\frac{P\left[s^{\prime}(r), s\right]}{N_{s^{\prime}}}+\Delta(r) \frac{P\left[s^{\prime}(r), s\right]}{P\left(s^{\prime}\right)}
$$

is a properly defined distribution, since it can be shown to be normalized and non-negative. The term in the right of Eq. (14) is the Kullback-Leibler divergence (Kullback 1968) between the distributions $P$ and $Q$, which is guaranteed to be non negative. This confirms the intuitive result $I_{D} \leq I$, the equality being only valid when

$$
\Delta(r) P\left[s^{\prime}(r), s\right]=\Delta(r, s) P\left[s^{\prime}(r)\right]
$$

for all $r$ and $s$.

Equation (14) states the quantitative difference between the full and the decoded information, and is the main result of this letter. The amount of lost information is therefore equal to the informational distance between the original probability distribution $P(r, s)$ and a new function $Q(r, s)$. It can be easily verified that

$$
I_{D}=\sum_{s} \sum_{r} Q(r, s) \log _{2}\left[\frac{Q(r, s)}{Q(r) Q(s)}\right]
$$

where

$$
\begin{aligned}
& Q(r)=\sum_{s} Q(r, s)=P(s), \\
& Q(s)=\sum_{r} Q(r, s)=P(r) .
\end{aligned}
$$

Therefore, the decoded information can be interpreted as a full mutual information between the stimuli and the responses, but with a distorted probability distribution $Q(r, s)$. In this context, the difference $I-I_{D}$ is no more than the distance between the true distribution $P(r, s)$ and the distorted one $Q(r, s)$.

When is Eq. 116 fulfilled? Surely, if there is at most one response in each class, $\Delta$ is always zero, and $I=I_{D}$. Also, if $P(r, s)$ is already flat in each class, there is no information loss. However, if $P(r, s)$ is not flat inside every class, but obeys the condition $P(r, s)=P_{s^{\prime}}(r) P\left(s^{\prime}, s\right)$ for a suitable $P\left(s^{\prime}, s\right)$ and some function $P_{s^{\prime}}(r)$ that sums up to unity within $\mathcal{C}\left(s^{\prime}\right)$, one can easily show that Eq. 16. holds. Just notice that this case implies that if $r_{1}$ and $r_{2}$ belong to $\mathcal{C}\left(s^{\prime}\right)$, then $P\left(r_{1}, s\right) / P\left(r_{2}, s\right)$ is independent of $r$, for all $s$. In other words, within each class $\mathcal{C}\left(s^{\prime}\right)$, the different functions $P(r \mid s)$ obtained by varying $s$ differ from one another by a multiplicative constant. These conditions coincide with the ones given by Panzeri et al. (1999) for having an exact decoding, within the short time limit. However, in the present derivation there are no assumptions about the interval in which responses are measured. Therefore, the decoding being exact whenever Eq. (16) is fulfilled is not a consequence of the short time limit carried out by Panzeri et al. (1999), but rather, a general property of the maximum likelihood decoding.
Next, by making a second order Taylor expansion of Eq. (14) in the distorsions $\Delta(r, s)$ and $\Delta(r)$ one may show that

$$
I=I_{D}+\sum_{s} \sum_{s^{\prime}} P\left(s^{\prime}, s\right) \frac{E\left(s^{\prime}, s\right)}{2 \ln 2}+\mathcal{O}\left(\Delta^{2}\right),
$$

where

$$
E\left(s^{\prime}, s\right)=\frac{1}{N_{s^{\prime}}} \sum_{r \in \mathcal{C}\left(s^{\prime}\right)}\left[\left(\frac{\Delta(r, s)}{P\left(s^{\prime}, s\right) / N_{s^{\prime}}}\right)^{2}-\left(\frac{\Delta(r)}{P\left(s^{\prime}\right) / N_{s^{\prime}}}\right)^{2}\right]
$$

Therefore, in the small $\Delta$ limit, the difference between $I$ and $I_{D}$ is quadratic in the distortions $\Delta(r, s)$ and $\Delta(r)$. This means that if in a given situation these quantities are guaranteed to be small, then the decoded information will be a good estimate of the full information. Equation (20) is equivalent to

$$
E\left(s^{\prime}, s\right)=\left\langle\left(\frac{P(r, s)}{P\left(s^{\prime}, s\right) / N_{s^{\prime}}}\right)^{2}-\left(\frac{P(r)}{P\left(s^{\prime}\right) / N_{s^{\prime}}}\right)^{2}\right\rangle_{\mathcal{C}\left(s^{\prime}\right)}
$$

where

$$
\langle f(r)\rangle_{\mathcal{C}\left(s^{\prime}\right)}=\frac{1}{N\left(s^{\prime}\right)} \sum_{r \in \mathcal{C}\left(s^{\prime}\right)} f(r)
$$

As a consequence, the relevant parameter in determining the size of $E\left(s^{\prime}, s\right)$ is given by the mean valuewithin $\mathcal{C}\left(s^{\prime}\right)$ - of a function that essentially measures how different are the true probability distributions $P(r, s)$ and $P(r)$, from their flattened versions $P\left(s^{\prime}, s\right) / N_{s^{\prime}}$ and $P\left(s^{\prime}\right) / N_{s^{\prime}}$.

To summarize, this letter presents the maximum likelihood decoding as an artificial-but useful-distortion of the distribution $P(r, s)$ within each class $\mathcal{C}\left(s^{\prime}\right)$. The decoded information is shown to be also a mutual information, the latter calculated with the distorted probability distribution. The difference between $I$ and $I_{D}$ is the Kullbach-Leibler distance between the true and distorted distributions. As such, it is always non negative, and it is easy to identify the conditions for the equality between the two information measures. Finally, for small distortions $\Delta$, the amount of lost information is expressed as a quadratic function in $\Delta$. In short, the aim of the work is to present a formal way of quantizing the effect of an optimal maximum likelihood decoding.

It should be kept in mind that in real situations, where only a limited amount of data is available, the estimation of $P(r \mid s)$ may well involve a careful analysis in itself. Some kind of assumption (as for example, a Gaussian shaped response variability) is usually required. The validity of the assumptions made depend on the particular data at hand. An inadequate choice for $P(r \mid s)$ may of course lead to a distorted value of $I$, and in fact, the bias 
may be in either direction. If the choice of $P(r \mid s)$ does not even allow the correct identification of the maximum likelihood stimulus (see Eq. (5)), then the calculated value of $I_{D}$ will also be distorted. The purpose of this letter, however, is to quantify how much information is lost when passing from $r$ to $s^{\prime}(r)$. No attempt has been made to quantify $I$ or $I_{D}$, for different estimations of $P(r \mid s)$.

Sometimes, $P\left(s^{\prime}, s\right)$ is defined in terms of $P(r, s)$ without actually decoding the stimulus to be associated to each response. For example, $P\left(s^{\prime}, s\right)$ can be introduced as $\sum_{r} P\left(r, s^{\prime}\right) P(r, s) / P^{2}(r)$ (Treves, 1997). This approach, although formally sound, is not based in a $r \rightarrow s^{\prime}$ mapping, and does not allow a partition of $\mathcal{R}$ into classes. It is therefore is not directly related to the analysis presented here. However, there might be analogous derivations where one may get to quantify the information loss also in this case.

\section{Acknowledgements}

I thank Bill Bialek, Anna Montagnini and Alessandro Treves for very useful discussions. This work has been partially supported with a grant of Proff. Treves, of the Human Frontier Science Program, number RG 01101998B.

\section{References}

- Bialek, W., Rieke, F., de Ruyter van Steveninck, R. R., \& Warland, D. (1991). Reading a neural code. Science, 252, 1854 - 1857.

- Cover, M. T., \& Thomas, J. A., (1991). Elements of Information Theory. New York: Wiley.

- Eskandar, E. N., Richmond, B. J., \& Optican, L., M. (1992). Role of inferior temporal neurons in visual memory. I. Temporal encoding of information about visual images, recalled images, and behavioural context. J. Neurophysiol., 68, 1277 1295 .

- Fano, R. M. (1961) Transmission of Information: A Statistical Theory of Communications. New York: MIT.

- Georgopoulos, A. P., Schwartz, A., \& Kettner, R. E. (1986). Neural population coding of movement direction. Science, 233, 1416 - 1419.

- Gochin, P. M., Colombo, M., Dorfman, G. A., Gerstein, G. L., \& Gross, C. G. (1994). Neural ensemble encoding in inferior temporal cortex. J. Neurophysiol, 71, 2325 - 2337.
- Golomb, D., Hertz, J., Panzeri, S., Treves, A., \& Richmond, B. (1997). How well can we estimate the information carried in neuronal responses from limited samples? Neural Comp., 9, 649 - 655.

- Heller, J., Hertz, J. A., Kjaer, T. W., \& Richmond, B. J. (1995). Information flow and temporal coding in primate pattern vision. J. Comput. Neurosci., 2, 175 - 193 .

- Kjaer, T. W., Hertz, J. A., \& Richmond, B. J. (1994). Decoding cortical neuronal signals: networks models, information estimation and spatial tuning. J. Comput. Neurosci., 1, 109 - 139.

- Kullback, S., (1968). Information theory and statistics. New York: Dover.

- Optican, L. M., \& Richmond, B. J. (1987). Temporal encoding of two dimensional patterns by single units in primate inferior temporal cortex: III Information theoretic analysis. J. Neurophysiol., 57, $162-178$.

- Panzeri, S., \& Treves, A. (1996). Analytical estimates of limited sampling biases in different information measures. Network, 7, 87 - 107.

- Panzeri, S., Treves, A., Schultz, S., \& Rolls, E. T. (1999). On decoding the responses of a population of neurons from short time windows. Neural Comput., 11, 1553 - 1577.

- Rieke, R., Warland, D., de Ruyter van Steveninck, R. R., \& Bialek W., (1996). Spikes: Exporing the Neural Code. Cambridge: MIT Press.

- Rolls, E. T., Critchley, H. D., \& Treves, A. (1996). Representation of Olfactory Information in the Primate Orbitofronal Cortex. J. Neurophysiol., 75, (5), 1982 - 1996.

- Rolls, E. T., Treves, A., \& Tovée, M. J. (1997). The representational capacity of the distributed encoding of information provided by populations of neurons in primate temporal visual area. Exp. Brain. Res., 114, 149 - 162.

- Rolls, E. T., \& Treves, A. (1998). Neural Networks and Brain Function. Oxford: Oxford University Press.

- Rolls, E. T., Treves, A., Robertson, R. G., GeorgesFrancois, P., \& Panzeri, S. (1998). Information About Spatial View in an Ensemble of Primate Hippocampal Cells. J. Neurophysiol., 79, 1797 - 1813.

- de Ruyter van Steveninck, R. R., \& Laughlin, S. B. (1996). The rates of information transfer at gradedpotential synapses. Nature, 379, 642 - 645. 
- Seung, H. S., \& Sompolinsky, H. (1993). Simple models for reading neural population codes. Proc. Nac. Ac. Sci. USA, 90, 10749 - 10753.

- Shannon, C. E. (1948). ATET Bell Laboratories Technical Jounal ,27, 379 - 423.

- Tovée, M. J., Rolls, E. T., Treves, A. \& Bellis, R. J. (1993). Information encoding and the responses of single neurons in the primate temporal visual cortex. J. Neurophysiol., 70, 640 - 654.

- Treves, A., \& Panzeri, S. (1995). The upward bias in measures of information derived from limited data samples. Neural Comp., 7, 399 - 407.

- Treves, A., Skaggs, W. E., \& Barnes, C. A. (1996). How much of the hippocampus can be explained by functional constraints? Hippocampus, 6, 666 - 674 .

- Treves, A. (1997). On the perceptual structure of face space. BioSyst., 40, 189 - 196.

- Victor, J. D., \& Purpura, K. P. (1996). Nature and precision of temporal coding in visual cortex: a metric space analysis. J. Neurophysiol., 76, 1310 $-1326$.

- Victor, J. D., \& Purpura, K. P. (1997). Metricspace analysis of spike trains: theory, algorithms and application. Network 8127 - 164

- Wilson, M. A., \& McNaughton, B. L. (1993). Dynamics of the Hippocampal Ensemble Code for Space. Science, 261, 1055 - 1058.

* Electronic address: samengo@cab.cnea.gov.ar

[1] It should be kept in mind, however, that when $I_{D}$ is calculated from actual recordings, its value is typically overestimated, because of limited sampling. Therefore, when dealing with real data sets, one may eventually obtain a value for $I_{D}$ that surpasses the true mutual information I. Nevertheless, whenever the number of elements in $\mathcal{S}^{\prime}$ is significantly smaller than the number of responses $r$, the sampling bias in $I_{D}$ will be bound by the one obtained in the estimation of $I$. 